\title{
Schizophyllum commune-derived $\beta$-glucan improves intestinal health demonstrating protective effects against constipation and common metabolic disorders
}

\author{
Vuong Vư ${ }^{1}$, Karthika Muthuramalingam², Vineet Singh ${ }^{3}$, Changmin $\mathrm{Choi}^{2}$, Young Mee Kim²,
} Tatsuya Unno ${ }^{3,4^{*}}$ and Moonjae Cho $1,25^{*}$

\begin{abstract}
It has been proven that $\beta$-glucan produced by Schizophyllum commune has beneficial effects on obesity, obesityassociated constipation, and colitis conditions; however, the protective effect of the compound on host at basal state is yet to be investigated. C57BL/6 J mice were fed with a normal diet (ND), normal diet supplemented with $3 \mathrm{~g} / \mathrm{kg}$ (BG_low), and $5 \mathrm{~g} / \mathrm{kg}$ (BG_high) of $\beta$-glucan (BG) for 12 weeks. Body weight, food and water intake and fecal status were monitored weekly. Intestine was stained by Periodic acid-Schiff (PAS) and Alcian Blue to evaluate the mucin layer thickness and goblet cell population. Morphological changes in internal organs and intestinal motility were also assessed, while serum biomarkers for liver injury and glucose level were analyzed. On the other hand, fecal microbiota and associated metabolic activities were also investigated. $\beta$-glucan bulked feces, decreased fecal moisture, and proliferated goblet cells resulted in a thickened lubricating mucin layer; however, the intestinal transit rate did not increase. The abundance of beneficial bacteria was increased while the harmful strains was decreased in a dose-dependent manner by the effect of $\beta$-glucan. Specific short chain fatty acid (SCFA)-producing strains, such as Roseburia, Ruminococcus, and Bifidobacteria, were selectively increased by $\beta$-glucan. In addition, consumption of $\beta$-glucan lowered level of obesity-associated biomarkers. Schizophyllum commune $\beta$-glucan showed an insignificant change in transit rate in healthy conditions when compared with obesity, despite similar effects on increasing mucus production and bulked feces. Nonetheless, the outcomes proposed protective effects against obesity, diabetes, inflammatory bowel diseases (IBD), and constipation, in which the modification of the gut microbiota by $\beta$-glucan is the largest contributor.
\end{abstract}

Keywords: $\beta$-glucan, Constipation, Gut microbiota, Intestinal epithelium, Obesity, Prebiotic

\section{Introduction}

Chronic constipation comprises functional constipation (FC) and constipation-type irritable bowel syndrome [1]. Infrequent and slow bowel movements are the two major

\footnotetext{
*Correspondence: tatsu@jejunu.ac.kr; moonjcho@jejunu.ac.kr

1 Interdisciplinary Graduate Program in Advanced Convergence

Technology \& Science, Jeju National University, Jeju 63241, Republic of Korea

${ }^{3}$ Faculty of Biotechnology, School of Life Sciences, SARI, Jeju National

University, Jeju 63243, Republic of Korea

Full list of author information is available at the end of the article
}

conditions in functional constipation [2]. The multifactorial pathogenesis of constipation consists of dietary habits, genetic factors, colonic absorption, motility, and medication history [2]. Furthermore, unhealthy lifestyle habits, such as lack of fiber intake, high consumption of dairy products, dehydration, and a sedentary lifestyle could also lead to unsatisfactory defecation [2, 3]. Following constipation, patients usually meet two or more of the following symptoms: excessive straining needed during defecation; lumpy and hard stool scale 1-2 on the Bristol Stool Scale; sensation of incomplete evacuation and 
anorectal obstruction/blockage; manual maneuvers, such as digital evacuation and support of pelvic floor needed; and fewer than three bowel movements per week [4].

It has been demonstrated that colonic mucus is decreased in rats with loperamide-induced constipation, while the consumption of both water-soluble and -insoluble fibers could relieve this condition [5, 6]. Importantly, different types of fiber lead to different results; for example, a $1.5 \mathrm{mg} /$ day administration of carrageenan and chondroitin sulfate or $5 \mathrm{mg} /$ day of sodium alginate increased fecal exertion, mucin production, and mucous layer thickness after 2 days, while administration of cellulose at $5 \mathrm{mg} /$ day was ineffective in mucin production [6]. Different effects of various fiber sources suggest that there are various beneficial fibers and effects that are yet to be thoroughly discovered. The composition of fecal and mucosal microbiota in patients with constipation is different from that in healthy individuals, suggesting a link between the gut microbiota and constipation [7]. It has been demonstrated that the population of Bacteroidetes is increased in the colonic mucosa of patients with constipation [7]. In addition, genera of Firmicutes, such as Faecalibacterium, Lactococcus, and Roseburia, correlated with fast colonic transit [7]. Previous reviews and meta-analyses have demonstrated that low levels of Lactobacillus spp. and high levels of Bifidobacterium, Clostridia, Bacteroides spp., Parabacteroides spp., and Proteus mirabilis were observed in children with functional constipation [8]. A meta-analysis demonstrated that the relationship between the gut microbiota and functional constipation is inconsistent, and no consensus exists [1]. However, low levels of Bifidobacterium in fecal samples and high levels of Bacteroidetes in the mucosa were observed in patients with irritable bowel syndromeconstipation [1]. The review also suggests that treatment with probiotics, such as Lactobacillus spp. and Bifidobacterium spp., prebiotics, synbiotics, and fecal microbiota transplantation are effective for treating chronic constipation with insignificant side effects $[1,8]$.

There is a wide range of treatments for constipation, and a medical approach using stimulant laxatives and osmotic could be used as the first strategy for patients with chronic constipation. If laxatives are ineffective, the use of lubiprostone, linaclotide, and prucalopride has also been suggested [2]. In addition, physiotherapy training of pelvic floor muscles and biofeedback therapy are especially useful for improving bowel symptoms, and in $70 \%$ of patients with gastrointestinal disorders, related symptoms have been eliminated using biofeedback therapy [2]. When other approaches are insufficient, surgical interventions can be applied [2]. Since therapies could be administered based on the mentioned etiologies, avoiding dehydration and increasing ingestion of fiber are the most reasonable and accessible treatments [3]. The clinical guideline/ position papers published by the North American Society for Pediatric Gastroenterology, Hepatology, and Nutrition (NASPGHAN) and the European Society of Pediatric Gastroenterology, Hepatology, and Nutrition (ESPGHAN) did not support the ingestion of fiber as a sufficient therapy for FC [9]. However, $86 \%$ of gastroenterologists from Europe and $81 \%$ from the US commonly recommend increasing the amount of fiber ingestion as a daily habit to amend FC [3]. In addition, previous meta-analyses showed that fiber ingestion resulted in an increased frequency of defecation, stool softness, and faster transit rate [9]. Together, this suggests that the use of fibers is still controversial, and further research is required. Previous studies have identified the effects of many types of fiber on FC and gastrointestinal function, including inulin [10], partially hydrolyzed guar gum [11], lactulose [12], glucomannan [13, 14], galacto-oligosaccharides [15], psyllium, and ispaghula [16]. In addition to these fibers, $\beta$-glucan has also been proposed as a beneficial prebiotic, an animal study showed that bread yeast $\beta$-glucan enhanced intestinal motility and recovered intestinal microecology, resulting in an increase in neurotransmitter and tight junction protein expression on loperamide-induced constipation [17]. Importantly, different sources and different molecular weights of $\beta$-glucan have distinct effects on the gastrointestinal tract, which suggests that the benefits of this compound have not been fully discovered [18-20]. Soluble and non-digestible $(1,3) /$ $(1,6)$ - $\beta$-glucan produced from Schizophyllum commune was recently shown to have an effect on high-fat dietinduced gut dysbiosis and ameliorated obesity-related constipation [21, 22]. Since the gut dysbiosis plays an important role in the progression of metabolic disorders, such as obesity, diabetes, and cardiovascular disease, the reformation of gut microbiota by this compound suggests protective effects against these diseases $[23,24]$. Furthermore, administration of Schizophyllum commune $\beta$-glucan fostered the fiber fermentation process and increased short chain fatty acids (SCFAs), which are metabolites known to fuel intestinal epithelial cells $[25,26]$.

In this study, the effects of Schizophyllum communederived $\beta$-glucan on the gastrointestinal tract on basal state were evaluated, focusing on physical changes, gut microbiota modification, modifying intestinal mucin production, gastrointestinal transit rate, and regulating associated serum biomarkers. Based on the acquired results, we indicate that the benefits of this compound on conditions such as constipation and common metabolic diseases could be observed even in the basal state. 


\section{Methods}

\section{Animal and animal care}

In the present study, 5-week-old male C57BL/6 J mice were used as a target for the treatment of $\beta$-glucan. Animal nourishment and handling in this experiment were compliant with the guidelines framed by the Animal Care and Use Committee (ACUC No.:2018-0018) at Jeju National University. Immediately after arriving at the laboratory, the mice were acclimatized to the experimental environment in the animal room with a $12 \mathrm{~h}$ light/dark cycle. The room temperature was maintained constant during the experiment at $23 \pm 2{ }^{\circ} \mathrm{C}$ with a humidity of $55 \pm 15 \%$. After 1 week of acclimatization, mice were randomly divided into three groups $(n=11$ per group): normal diet (ND), ND supplemented with $\beta$-glucan at a concentration of $3 \mathrm{~g} / \mathrm{kg}$ (BG_low), and $5 \mathrm{~g} / \mathrm{kg}$ (BG_high). BG_low and BG_high were prepared by directly adding $\beta$-glucan to the normal diet ingredients. Pharmacologically active and purified $\beta$-glucan produced from Schizophyllum commune were provided by Quegen Biotech Co. Ltd. (Seoul, Republic of Korea). The soluble $\beta-1,6$ branching $\beta$-1,3-glucan compound has a molecular weight of $1.78-1.79 \times 106 \mathrm{Da}$ and a purity of $95 \%$. During the animal experiment, mice were housed with ad libitum access to food and water, and animal food was replaced every day, while the total consumption was monitored weekly.

\section{Physiological measurement}

Animal body weight and food and water consumption were monitored weekly throughout the study. Wooden chips were used as animal bedding, the bedding was replaced every week. At $24 \mathrm{~h}$ after bedding replacement, 25 pieces of feces from each cage were collected for weighing. Photographs of the feces were also taken for color, size, and morphology evaluation. After 12 weeks of animal study, mice were sacrificed and tissues such as the small intestine, colon, liver, and lung were collected and stored at $-70{ }^{\circ} \mathrm{C}$. The distance from the duodenum to the terminal ileum (small intestine) and the distance from the cecum to the rectum (colon) were measured. The liver and lung weights were also evaluated, the liver and lung indices were calculated using the following formula: liver index $(\%)=$ liver weight $(\mathrm{g}) /$ body weight $(\mathrm{g}) \times 100(\%)$. The increased percentage of body weight was calculated using the following formula: increased body weight $(\%)=12$ th body weight $(\mathrm{g}) /$ initial body weight $(\mathrm{g}) \times 100$.

\section{Fecal moisture content analysis}

Equal amounts of feces were collected from each group and kept in a hot air oven at $60{ }^{\circ} \mathrm{C}$ for 2 days to measure dry weight. The moisture content was calculated by subtracting the dry weight from the wet weight. Moisture content $=($ wet weight - dry weight $) /$ wet weight $\times 100$.

\section{Feed efficiency calculation}

Body weight and food consumption were monitored weekly. Feed efficiency was calculated as follows: feed efficiency $(\%)=$ increased body weight $(\mathrm{g}) /$ total food intake $(\mathrm{g}) \times 100$.

\section{Fecal transit rate}

The intestinal mobility rate was calculated using activated charcoal $(100 \mu \mathrm{L})$. The mice were starved overnight and were administered with activated charcoal $20 \mathrm{~min}$ before sacrifice. The length of the small intestine and the distance travelled by charcoal were measured. The transit rate was calculated as follows:

Intestinal transit rate $(\%)=$ charcoal travelled distance $(\mathrm{cm}) /$ small intestinal length $(\mathrm{cm}) \times 100$.

\section{Blood profiling}

Immediately before sacrifice, blood was drawn from each mouse. Serum was collected by centrifuging blood at $500 \times g$ for $10 \mathrm{~min}$, and analyzed by ChemOn Inc. (Republic of Korea) to test biomarkers associated with liver damage and overall energy homeostasis, such as aspartate transaminase (AST), alanine transaminase (ALT), alkaline phosphatase (ALP), glucose (GLU), lactate dehydrogenase (LDH), and high-density lipoprotein (HDL). A clinical biochemical analyzer (AU680, Beckman Coulter, Japan) was used to analyze these parameters.

\section{Histopathological analysis}

The small intestinal samples were sectioned and subjected to periodic acid-Schiff (PAS) and Alcian blue staining. Images of the histological samples were taken using an Olympus BX51 microscope. ImageJ software was used for histological analysis, and the mucus thickness layer was evaluated using stained intestine images (ten measurements per section per animal, four animals per group; mucus layers include mucosal and submucosal layers).

\section{Fecal microbiota analysis}

Fecal microbiota analysis was described in previous study [26]. Deoxyribonucleic acid (DNA) was extracted from the mouse fecal samples using the QIAamp PowerFecal Pro DNA Kit (QIAamp, USA). The V3-4 regions of the $16 \mathrm{~S}$ rRNA gene were amplified using region-specific primers $341 \mathrm{~F}$ and $806 \mathrm{R}$. The MiSeq library was prepared according to the protocol provided by Illumina Inc. (USA). MiSeq sequencing was performed at Macrogen 
(Seoul, South Korea) according to the manufacturer's instructions. Mothur was used for MiSeq data analysis [27]. Briefly, the MiSeq output was first paired-end assembled and then aligned to Silva_v138 [28] using the align.seqs. Low-quality reads, such as ambiguous base calls and lengths not between 400 and $600 \mathrm{bp}$, were removed. The pre.cluster Mothur subroutine was applied to correct the machinery errors. Chimeric sequences were detected using VSEARCH [29] and removed using the remove.seqs Mothur subroutine. Taxonomy classification was performed using the Ribosomal Database Project (RDP) trainset18 [30] and classify.seqs Mothur subroutine. The mitochondrial or chloroplast sequences were removed. Operational taxonomic units (OTUs) were determined using the OptiClust [31], and were used to calculate the Bray-Curtis distances. Non-metric multidimensional scaling (NMDS) analysis was performed to estimate the microbial shifts among the groups. The differential abundance test was performed using the linear discriminant analysis effect size (LEfSe) [32] implemented in Mothur. PICRUSt2 was used to predict the intestinal metabolic activity [33]. STAMP [34] was used to identify differentially abundant metabolic activities with significance at $\mathrm{P}<0.05$, adjusted with BenjaminiHochber multiple test correction. Heatmaps were drawn using the heatmap. $2 \mathrm{R}$ package. Analysis of molecular variance (AMOVA) was used to estimate the significant differences in the NMDS plots.

\section{Statistical analysis}

Student's t-test was used to analyze significant differences in most experiments, and the difference with a p-value of less than 0.05 , was considered significant. The data in this paper are presented as the mean \pm SD.

\section{Result \\ $\beta$-glucan increased feed efficiency, fecal moist and changed fecal morphology}

After 12 weeks of treatment, the BG_high diet significantly increased body weight compared to the normal diet and BG_low treated groups (Fig. 1a). Both $\beta$-glucan treated groups showed high fecal weight throughout the 12-week feeding period, although not significant (Fig. 1b). Similar food intake is observed among the three groups, while mice in $\beta$-glucan treated groups tend to consume less water (Fig. 1c, d). Treatment of $5 \mathrm{~g} / \mathrm{kg}$ of $\beta$-glucan markedly increased feed efficiency compared to $3 \mathrm{~g} / \mathrm{kg}$ group (Fig. 1e). Concomitant with a decreased fecal moisture content in the $\beta$-glucan treated group, stools from $\beta$-glucan treated mice had larger size and lighter color compared to the normal diet and BG_low treated groups (Fig. 1f, g).

\section{$\beta$-glucan proliferated goblet cells and increased mucin production}

Both BG_low and BG_high diets increased mucus secretion, which is displayed by the pink color area in PAS staining images and blue color area in Alcian blue staining images (Fig. 2a, b). The thickness of the mucosa layer was measured using PAS and Alcian blue staining images (Fig. 2c). Administration of high concentrations of $\beta$-glucan resulted in a significantly thicker mucosal layer (Fig. 2c). PAS staining of intracellular mucus clearly showed that the increase in mucin production is due to the induced proliferation of the goblet cells by $\beta$-glucan.

\section{$\beta$-glucan increased intestinal length and liver and lung weight}

$\beta$-glucan lengthened the small intestine and colon in the animals (Fig. 3a, b). In addition, consumption of $\beta$-glucan increased the weight of the liver and lungs (Fig. 3c, d). Measurement of intestinal motility using activated carbon showed that $\beta$-glucan did not improve the intestinal transit rate (data not shown); however, our previous study using a high-fat diet as a control, HFD supplemented with $\beta$-glucan, showed a significant increase in the gastrointestinal transit rate [21]. Blood profiling assessment demonstrated that $\beta$-glucan significantly decreased the level of liver injury biomarkers, such as AST, ALT, and ALP, in a dose-dependent manner (Table 1). In addition, glucose levels were significantly low in the BG-treated groups, especially in the BG_high group. LDH and HDL serum levels were upregulated in the $\beta$-glucan-treated group, with high levels in BG_high-treated mice (Table 1).

\section{$\beta$-glucan decreased harmful strains while selectively fostered SCFAs-producing strains}

In this study, we obtained 2,043,883 clean reads and 10,000 reads per sample were used for analyses. Alphadiversity analysis showed no difference between dietary groups, neither for species richness nor evenness (Additional file 1: Fig. S1). Taxonomic composition analysis showed a clear difference between ND and BG groups at the phylum level, where a high abundance of Verrucomicrobia is observed (Additional file 1: Fig. S2A). Distinct taxonomic compositions were not observed at the family and genus levels (Additional file 1: Fig. S2B, C). However, beta-diversity analysis showed significant microbiota differences among the groups $(\mathrm{P}<0.01)$ (Fig. 4a). Differential abundance tests at the genus level showed that Butyricicoccus, Parabacteroides, and Clostridium_IV were decreased by $\beta$-glucan treatment (Fig. 4b, c). The high concentration of $\beta$-glucan further decreased the abundance of the genus Akkermansia. In contrast, $\beta$-glucan treatment increased the abundance 


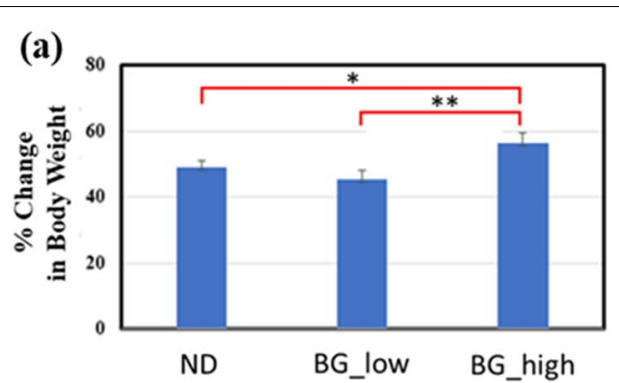

(c)

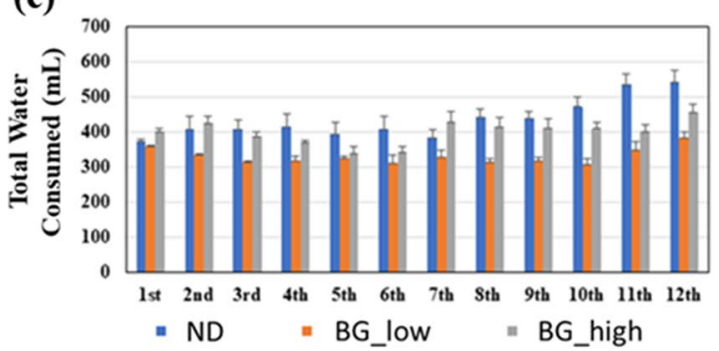

(e)

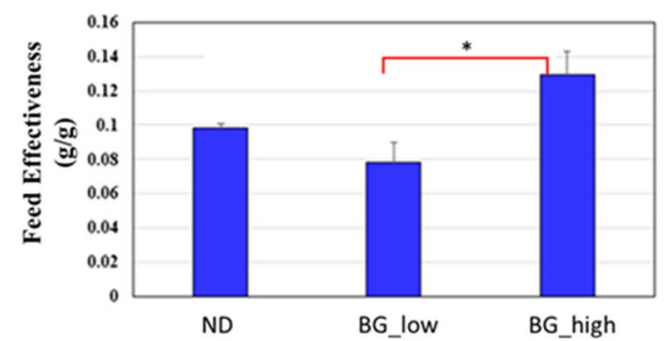

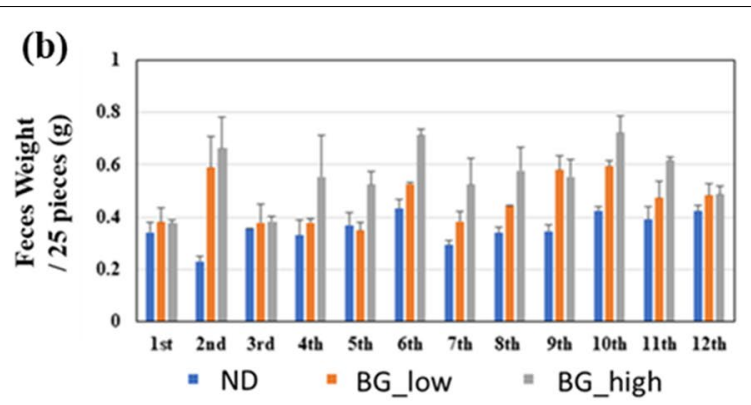

(d)
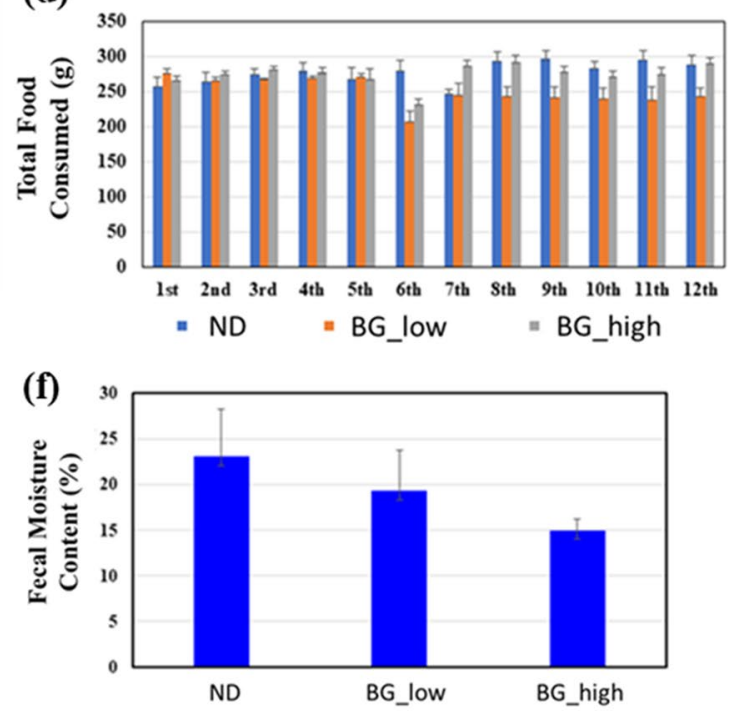

(g)

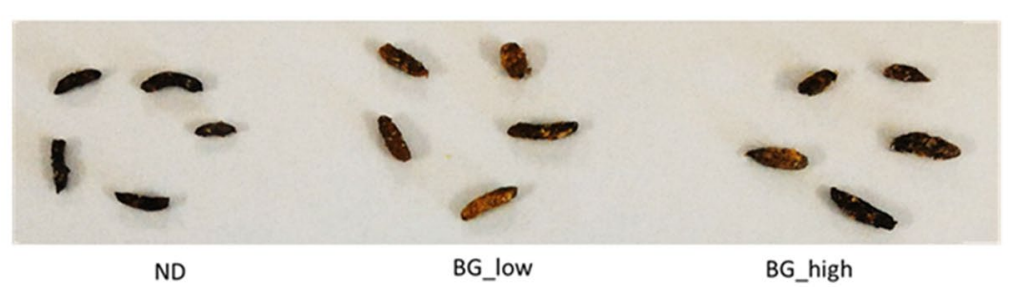

Fig. 1 Effect of $\beta$-glucan on food and water consumption and fecal status. After 12 weeks of feeding, several parameters are evaluated and compared among groups ( $n=11$ per group). a Body weight; $\mathbf{b}$ fecal weight; $\mathbf{c}$ total water consumed; $\mathbf{d}$ total food consumed; $\mathbf{e}$ feed effectiveness; $\mathbf{f}$ fecal moisture content; $\mathbf{g}$ fecal morphology. Statistically significant results are labeled as ${ }^{*} \mathrm{P}<0.05$; ${ }^{* *} \mathrm{P}<0.01$

of Bifidobacterium, Ruminococcus, and Roseburia. Metabolic activities predicted by the PICRUSt 2 showed that high concentrations of $\beta$-glucan significantly enriched and depleted 11 and 8 metabolisms, respectively. However, only four metabolic pathways were increased, and two pathways were decreased by low concentrations of $\beta$-glucan. However, hexitol degradation is the only metabolic activity with significant differential abundance between low and high concentrations of BG (Additional file 1: Fig. S3). Enriched metabolism due to high concentrations of $\beta$-glucan is mostly related to nucleotide degradation and amino acid biosynthesis, while depleted metabolism is related to menaquinol biosynthesis (Additional file 1: Fig. S3, Table 2).

\section{Discussion}

$\beta$-glucan showed protective effect against constipation and metabolic disorders

After 12 weeks of treatment, $3 \mathrm{~g} / \mathrm{kg}$ of Schizophyllum commune-derived $\beta$-glucan did not increase the animals' body weight; however, consumption of $5 \mathrm{~g} / \mathrm{kg} \beta$-glucan resulted in a significant increase in body weight and feed efficiency. Although it is widely known that consumption of fiber is related to lowered body weight [35], in 
(a) PAS staining

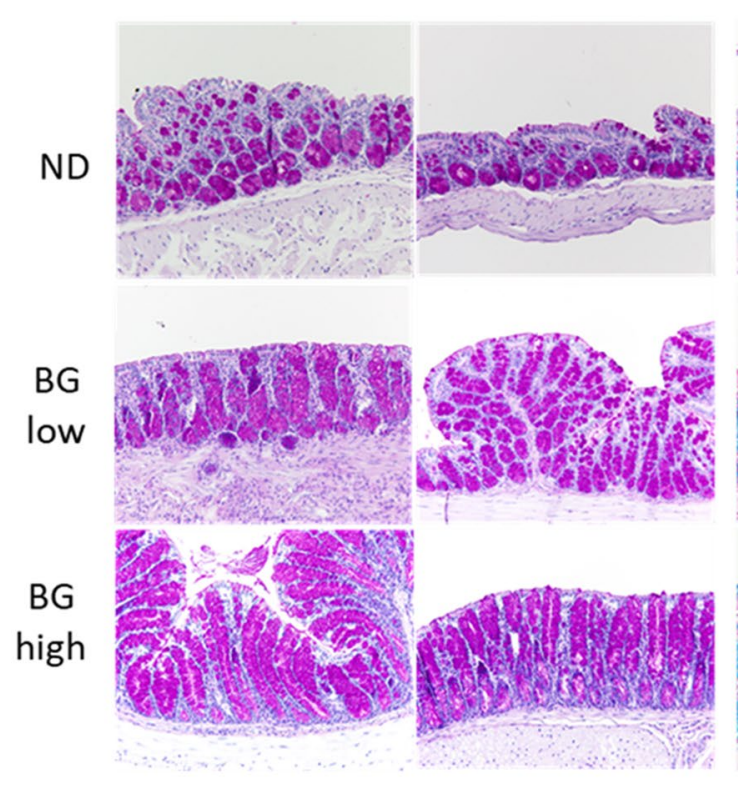

(b) Alcian Blue staining

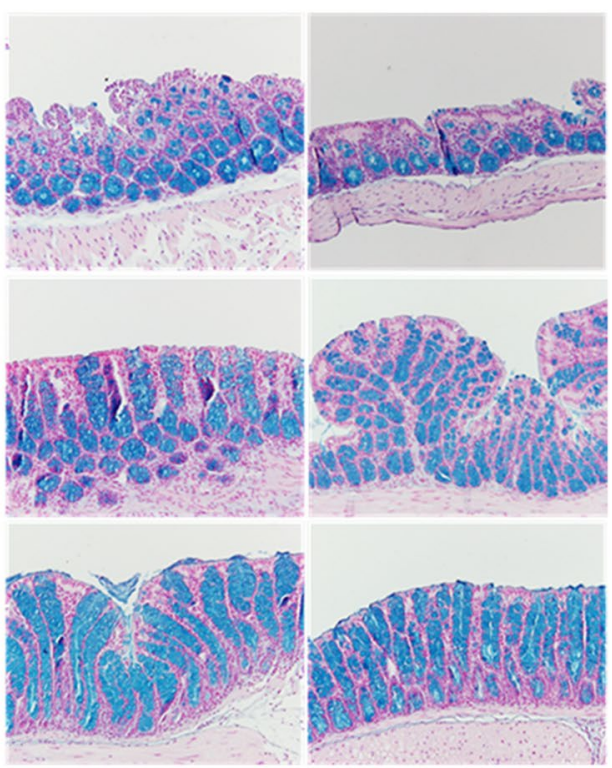

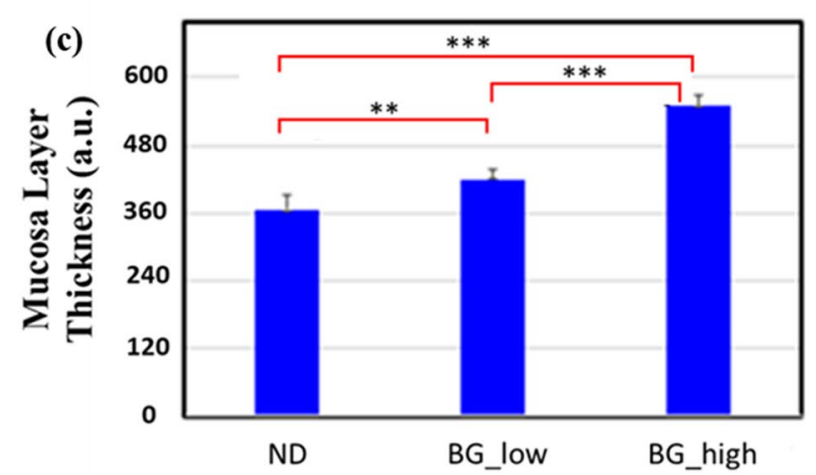

Fig. 2 Effect of $\beta$-glucan on mucus thickness and goblet cell proliferation. a The intracellular mucus of the goblet cells is stained using periodic acid-Schiff (PAS); $\mathbf{b}$ however, PAS could not stain the acidic mucus, which is stained by Alcian blue instead; $\mathbf{c}$ mucosa layer thickness was measured by applying ImageJ software to analyze sectioned intestine images. Statistically significant results are labeled as ${ }^{*} P<0.05 ;{ }^{* *} P<0.01 ; * * P<0.001$

the present study, consumption of Schizophyllum commune $\beta$-glucan increased not only the body weight, but also the lung and liver index significantly. An increase in body weight usually increases the risk of obesity and dysfunction of overall nutrition metabolism, followed by the upregulation of specific serum markers, such as AST, ALT, ALP, and cholesterol. Despite the increases in body weight and liver weight, biomarkers for liver injury, such as AST, ALT, and ALP were downregulated in both $\beta$-glucan-treated groups, suggesting that $\beta$-glucan has the potential to reduce the risk of liver damage. These results suggest a healthy weight gain by $\beta$-glucan and the protective effect of $\beta$-glucan against liver injury, which was also reported in a high-fat dietinduced obesity study [21]. Better nutrition metabolism and water absorption could explain the weight gain since our concurrent study showed that $\beta$-glucan successfully decreased HFD-induced weight gain and metabolic distress [21]. In addition, a significant decrease in the glucose level was observed, suggesting a protective effect against diabetes. HDL cholesterol is known as good cholesterol that has effect on cholesterol clearance, thus preventing atherosclerosis [36, 37], which was increased by $\beta$-glucan treatment. In contrast, low-density lipoprotein (LDL) cholesterol, also called bad cholesterol, was slightly decreased at high doses of $\beta$-glucan.

Together with an increase in BW, mice treated with $\beta$-glucan had high fecal weight, large size, and lightcolored feces; however, the fecal transit rate was not increased by $\beta$-glucan treatment. In comparison with 
(a)

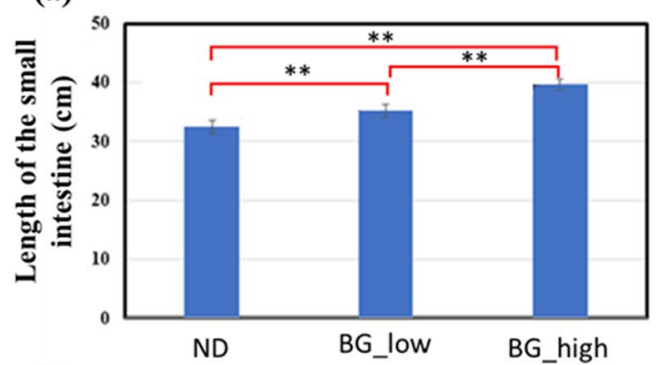

(c)

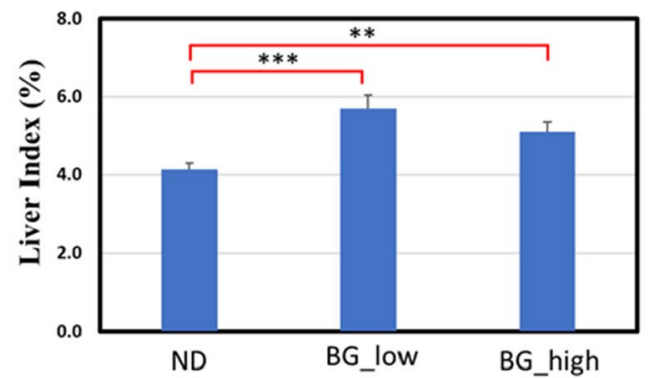

(b)

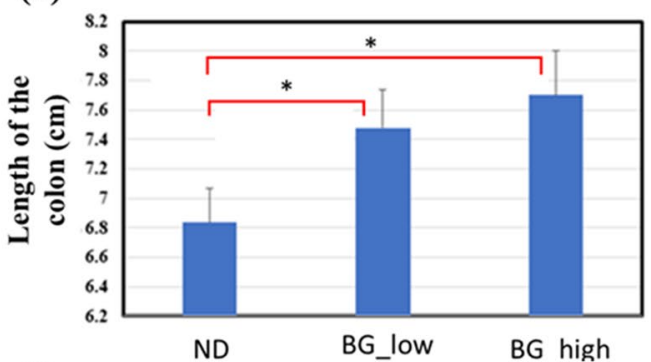

(d)

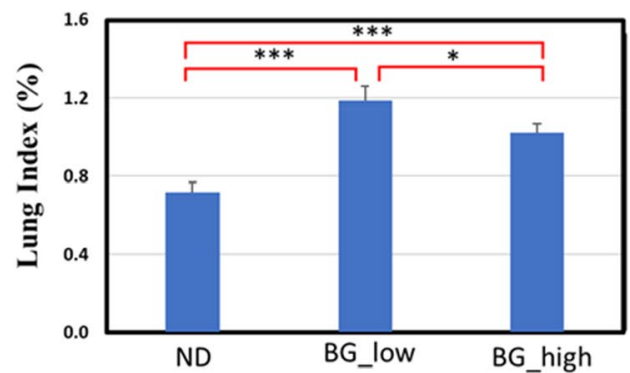

Fig. 3 Effect of $\beta$-glucan on anatomic changes in C57BL/6 J mice. The small intestine and colon of the mice are measured by a ruler, while the animal liver and lung are weighed. $\mathbf{a}$ small intestine length; $\mathbf{b}$ colon length; $\mathbf{c}$ Liver index; $\mathbf{d}$ Lung index. Statistically significant results are labeled as ${ }^{*} \mathrm{P}<0.05 ;{ }^{* *} \mathrm{P}<0.01 ;{ }^{* * *} \mathrm{P}<0.001$

Table 1 Blood profiling for serum markers of liver injury and cardiovascular disease

\begin{tabular}{lccc}
\hline Blood profiling & ND $(\boldsymbol{n}=\mathbf{1 1})$ & BG_low $(\boldsymbol{n}=\mathbf{1 1})$ & BG_high $(\boldsymbol{n}=\mathbf{1 1})$ \\
\hline AST (U/L) & $166.2 \pm 63.0$ & $164.6 \pm 58.3$ & $149.7 \pm 48.0$ \\
ALT (U/L) & $39.7 \pm 14.0$ & $36.6 \pm 4.3$ & $34.6 \pm 2.1$ \\
ALP (U/L) & $82.8 \pm 13.3$ & $81.1 \pm 6.2$ & $80.4 \pm 13.0$ \\
GLU (mg/dL) & $173.2 \pm 27.6$ & $121.9 \pm 26.6^{*}$ & $107.5 \pm 45.4^{*}$ \\
HDL (mg/dL) & $66.7 \pm 3.3$ & $83.5 \pm 6.9^{*}$ & $83.0 \pm 5.3^{*}$ \\
LDL (mg/dL) & $24.3 \pm 6.0$ & $24.2 \pm 1.1$ & $22 \pm 3.0$ \\
\hline
\end{tabular}

*Compared to normal diet: $\mathrm{P}<0.05$

our previous study using an HFD-fed model, mice in both normal and obese conditions showed bulked feces during $\beta$-glucan treatment, and the significantly increased transit rate was only observed in the obese mice [21]. In addition, mice that consumed $\beta$-glucan tended to drink less water, which resulted in a low moisture content. Controversially, it has been reported that statements such as bulking feces by fiber treatment can result in better bowel movement are illogical [38], however, a meta-analysis strongly stated that dietary fiber is a potential therapeutic compound that could be used to ameliorate constipation [39]. The interesting controversy about the effect of fiber on constipation symptoms raised the need to investigate the effect of a certain fiber on the host in both healthy and diseased conditions. Our results suggested that the effect of $\beta$-glucan could be varied due to many aspects, such as types of hosts (human or mouse), host conditions (healthy or obesity), dietary habits (normal diet and high-fat diet with low or high fiber), and most importantly the amount of water consumption. The results showed that $\beta$-glucan treatment under normal conditions did not lead to a significant decrease in body weight or intestinal transit rate, which was observed in obese conditions [21]. However, treatment with $\beta$-glucan successfully decreased obesity followed serum factors, induced better water absorption, bulked feces, proliferated goblet cell populations, and increased mucin production even under normal conditions, which implies a protective effect against obesity, diabetes, and constipation.

\section{$\beta$-glucan improved intestinal overall healthiness}

Administration of $\beta$-glucan increased mucin production, suggesting an important role of $\beta$-glucan in regulating the gut permeability and providing a lubricant that improves stool transition, implying the ability of $\beta$-glucan to relieve constipation. SCFAs, a product of fiber fermentation produced by gut microbiota, are important nutrients for intestinal epithelial cells that improve intestinal integrity and enhance mucin production [21, 25]. Increased production of SCFAs in mice was observed following the administration of $\beta$-glucan [26]. The increased 
(a)
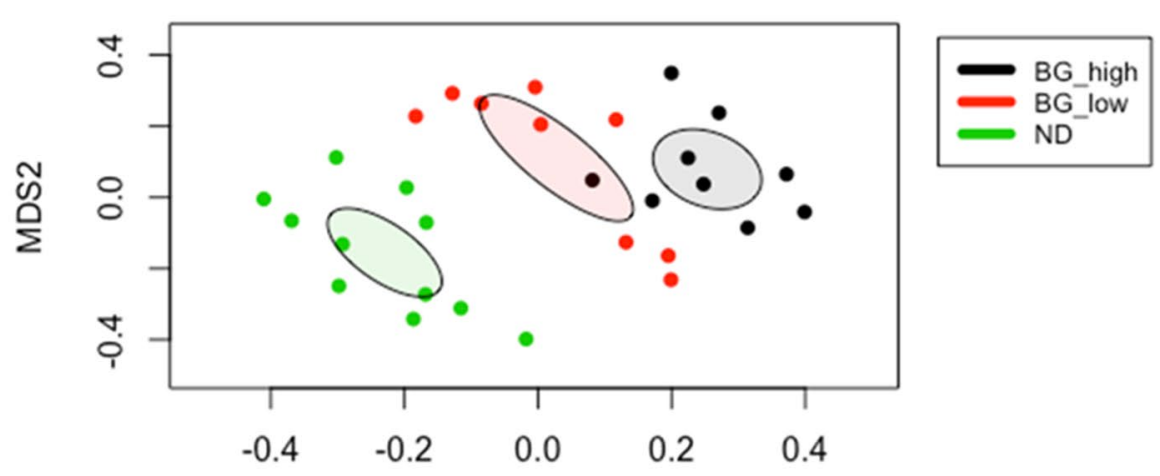

MDS1

\section{LEfSe}

(b)

$$
\begin{array}{r}
\text { Burkholderiales_unclassified } \\
\text { Butyricicoccus } \\
\text { Parabacteroides } \\
\text { Clostridium IV } \\
\text { Bilophila } \\
\text { Roseburia } \\
\text { Sporobacter } \\
\text { Anaeroplasma } \\
\text { Odoribacter } \\
\text { Ruminococcus } \\
\text { Brifidobacterium } \\
\text { Proteobacteria unclassied } \\
\text { Firmicutes_unclassified } \\
\text { Clostridiales_unclassified }
\end{array}
$$

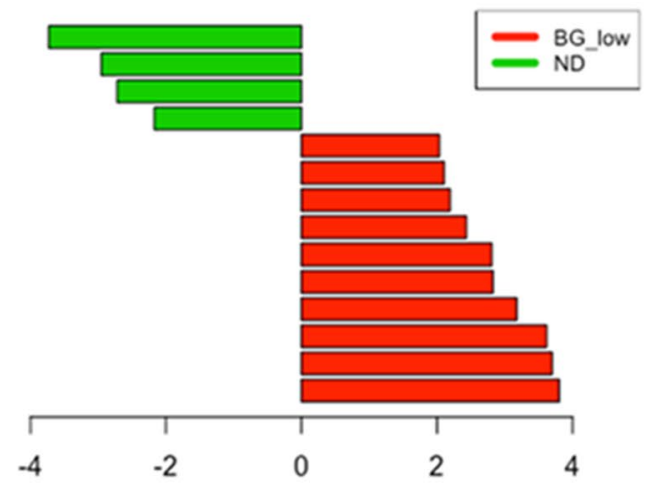

\section{LEfSe}

(c)
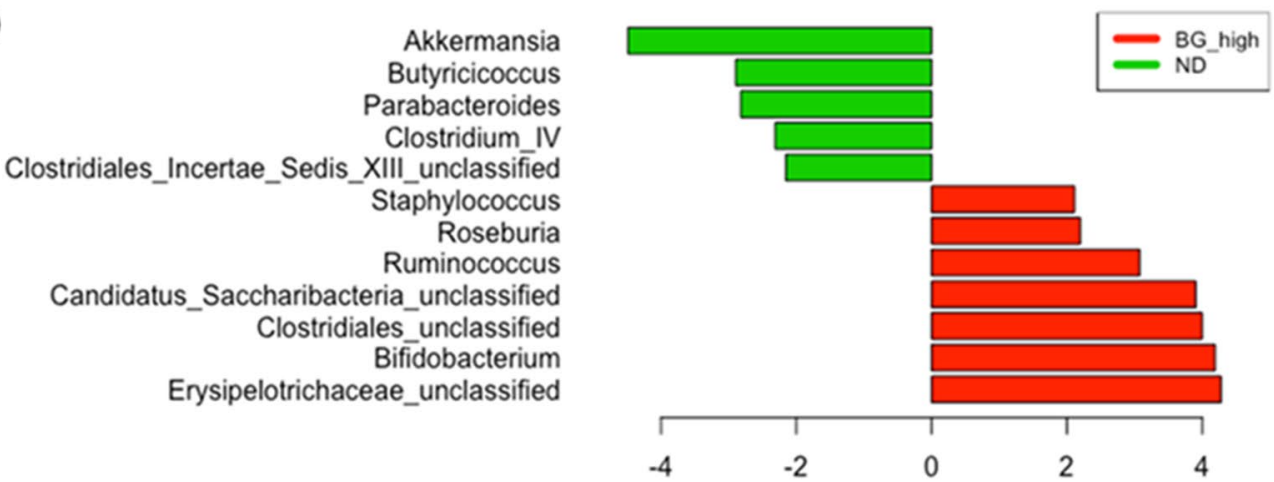

Fig. 4 Effect of $\beta$-glucan on microbiota modification in C57BL/6 J mice. a Beta-diversity comparison among the dietary groups through non-multidimensional scaling analysis; $\mathbf{b}$ differentially abundant genera between ND and BG_low; c ND and BG_high

mucus secretion mainly results from an increased number of mucus-secreting goblet cells. Our previous study clearly demonstrated that treatment with Schizophyllum commune-derived $\beta$-glucan increased proliferation and differentiation markers of intestinal cells such as cyclin D1, cyclin E, K14, p21, and p27 [21]. In this study, treatment with $\beta$-glucan significantly increased the length of the small intestine and colon. A longer gastrointestinal tract allows more absorption of water, which results in a lower moisture content in stools. In addition, treatment of high-fat diet incorporated with $\beta$-glucan on DSS-induced colitis showed increased expression level of tight-junction proteins (claudin-1) and adhesion proteins (E-cad) compared to consumption of a high-fat diet 
Table 2 Metabolic activity differences between ND and BG high (Effect size >0.1)

\begin{tabular}{|c|c|c|c|}
\hline Enriched group & Pathways & Descriptions & $\begin{array}{l}\text { Effect size } \\
\text { difference }\end{array}$ \\
\hline BG_high & SALVADEHYPOX-PWY & Adenosine nucleotides degradation II & 0.24 \\
\hline BG_high & PWY-6608 & Guanosine nucleotides degradation III & 0.22 \\
\hline BG_high & P161-PWY & Acetylene degradation & 0.15 \\
\hline BG_high & PWY-1861 & Formaldehyde assimilation II (RuMP Cycle) & 0.15 \\
\hline BG_high & PWY-5347 & Superpathway of L-methionine biosynthesis (transsulfuration) & 0.13 \\
\hline BG_high & PWY-6353 & Purine nucleotides degradation II (aerobic) & 0.13 \\
\hline BG_high & P4-PWY & Superpathway of L-lysine, L-threonine and L-methionine biosynthesis I & 0.12 \\
\hline BG_high & PWY-6901 & Superpathway of glucose and xylose degradation & 0.12 \\
\hline BG_high & PWY0-781 & Aspartate superpathway & 0.11 \\
\hline BG_high & MET-SAM-PWY & Superpathway of S-adenosyl-L-methionine biosynthesis & 0.11 \\
\hline BG_high & RUMP-PWY & Formaldehyde oxidation I & 0.10 \\
\hline ND & PWY-5838 & Superpathway of menaquinol-8 biosynthesis I & 0.17 \\
\hline ND & PWY-5840 & Superpathway of menaquinol-7 biosynthesis & 0.16 \\
\hline ND & PWY-5899 & Superpathway of menaquinol-13 biosynthesis & 0.16 \\
\hline ND & PWY-5897 & Superpathway of menaquinol-11 biosynthesis & 0.16 \\
\hline ND & PWY-7315 & dTDP-N-acetylthomosamine biosynthesis & 0.13 \\
\hline ND & PWY-5861 & Superpathway of demethylmenaquinol-8 biosynthesis & 0.13 \\
\hline ND & FASYN-ELONG-PWY & Fatty acid elongation—saturated & 0.11 \\
\hline ND & P108-PWY & Pyruvate fermentation to propanoate I & 0.10 \\
\hline
\end{tabular}

alone $[21,26]$. The population of healthy epithelial cells and increased expression of tight-junction proteins are essential for gut permeability and intestinal barrier regulation, which prevents transmural infiltration of bacteria and microbial lipopolysaccharide (LPS), causing inflammation of the intestine, liver, and adipose tissue $[24,40]$. These results suggest that $\beta$-glucan could protect the gut from IBD and IBD-induced non-alcoholic fatty liver disease (NAFLD), which was proven in our previous study on the effect of $\beta$-glucan on ulcerative colitis [26].

\section{Schizophyllum commune $\beta$-glucan shifted gut microbiota to the healthy population}

It has been proven that SCFAs are important metabolites produced via fiber fermentation by the gut microbiota, which fuels the intestinal epithelial cells, are increased under treatment with Schizophyllum commune $\beta$-glucan [26]. In the present study, consumption of $\beta$-glucan dosedependently supports beneficial gut microbiota, such as Anaerostipes, Roseburia, and Coprobacillus, which play roles in fermenting glucose to lactate and catabolizing lactate to produce butyrate [41-43]. In addition, the population of obesity-associated bacteria, such as Parabacteroides and Lactococcus is decreased by treatment with $\beta$-glucan, suggesting an anti-obesity effect of this compound [21]. $\beta$ glucan decreases many SCFA-producing bacteria, such as Butyricicoccus and Parabacteroides, and fiber degraders, such as Clostridium_IV and
Akkermansia, while significantly increases other SCFAproducing bacteria and probiotics, such as Roseburia, Ruminococcus, and Bifidobacteria. Therefore, it is likely that the abundance of these species changed due to the competition over nutrient availability in the gut; that is, $\beta$-glucan supplementation selectively increasess some species of SCFA-producing bacteria. $\beta$-glucan consumption suppresses the Akkermansia population, in which the strain is known to degrade intestinal mucin and lead to stool drying and hard defecation [44]. Comparison of metabolic activities indicated that higher concentrations of $\beta$-glucan affected intestinal/bacterial metabolic activities in the gut stronger than lower concentrations of $\beta$-glucan, although the two groups showed relatively similar metabolic activities. $\beta$-glucan increases degradation of purine nucleotides, such as adenosine and guanosine, the metabolism of which has been reported to increase the immune system in humans [45]. In contrast, $\beta$-glucan decreased menaquinol (reduced vitamin K2) biosynthesis, in which menaquinol-8 (PWY-5838) was reported to be positively associated with type 2 diabetes [46].

In conclusion, Schizophyllum commune $\beta$-glucan improved intestinal health by enhancing water absorption, proliferating epithelial cells, and increasing lubricating mucin production, which is essential for the treatment of constipation. Moreover, administration of $\beta$-glucan prevents the risk of conditions, such as obesity, diabetes, atherosclerosis, and intestinal inflammation diseases that 
are observed with reduction in serum glucose levels and biomarkers associated with liver injury, while upregulating HDL. Modification of the gut microbiota toward a healthy community due to the compound plays a major role in obtaining these benefits. The study consolidated the protective effect of Schizophyllum commune against various diseases in basal state; however, further studies using specific models are needed to comprehensibly conclude the effect of Schizophyllum commune $\beta$-glucan on these conditions.

\section{Supplementary Information}

The online version contains supplementary material available at https://doi. org/10.1186/s13765-022-00680-3.

Additional file 1: Figure S1. Alpha-diversity comparison among dietary groups for species richness (a) and evenness (b) estimated by Chao I and Shannon, respectively. Figure S2. Taxonomic composition analysis among dietary groups at the phylum (a), family (b), and genus levels (c). Figure S3. Metabolic activity differences between normal diet and BG low groups (a), BG low and BG high groups (b), and ND and BG high groups (c).

\section{Acknowledgements}

We would like to thank Editage (www.editage.co.kr) for English language editing.

\section{Authors' contributions}

W and KM collected and analyzed the data. WV drafted the manuscript. KM and CC performed experiments. VS took part in fecal microbiota analysis. SIC contributed the $\beta$-glucan. YMK planned and supervised the research. TU and MJC gave the research idea and designed the analysis and proofread the manuscript. All authors have read and approved the final manuscript.

\section{Funding}

This research was supported by the Basic Science Research Program through the National Research Foundation of Korea (NRF) funded by the Ministry of Education, Science, and Technology (NRF-2020R111A3072840).

\section{Availability of data and materials}

All data generated or analysed during this study are included in this published article (and its Additional file 1)

\section{Declarations}

\section{Competing interests}

The authors declare that they have no competing interests.

\section{Author details \\ ${ }_{1}^{1}$ Interdisciplinary Graduate Program in Advanced Convergence Technology \& Science, Jeju National University, Jeju 63241, Republic of Korea. ${ }^{2}$ Depart- ment of Biochemistry, School of Medicine, Jeju National University, Jeju 63243, Republic of Korea. ${ }^{3}$ Faculty of Biotechnology, School of Life Sciences, SARI, Jeju National University, Jeju 63243, Republic of Korea. ${ }^{4}$ Subtropical/Tropical Organism Gene Bank, Jeju National University, Jeju 63243, Republic of Korea. ${ }^{5}$ Institute of Medical Science, Jeju National University, Jeju 63241, Republic of Korea.}

Received: 14 November 2021 Accepted: 19 January 2022

Published online: 02 February 2022

\section{References}

1. Ohkusa T, Koido S, Nishikawa Y, Sato N (2019) Gut microbiota and chronic constipation: a review and update. Front Med (Lausanne) 6:19-19. https://doi.org/10.3389/fmed.2019.00019

2. Forootan M, Bagheri N, Darvishi M (2018) Chronic constipation: a review of literature. Medicine (Baltimore) 97:e10631-e10631. https://doi.org/10. 1097/MD.0000000000010631

3. Axelrod CH, Saps M (2018) The role of fiber in the treatment of functional gastrointestinal disorders in children. Nutrients 10:1650. https://doi.org/ 10.3390/nu10111650

4. Prichard DO, Bharucha AE (2018) Recent advances in understanding and managing chronic constipation. F1000Res 7:1640. https://doi.org/10. 12688/f1000research.15900.1

5. Shimotoyodome A, Meguro S, Hase T, Tokimitsu I, Sakata T (2000) Decreased colonic mucus in rats with loperamide-induced constipation. Comp Biochem Physiol A Mol Integr Physiol 126:203-212. https://doi.org/ 10.1016/S1095-6433(00)00194-X

6. Shimotoyodome A, Meguro S, Hase T, Tokimitsu I, Sakata T (2001) Sulfated polysaccharides, but not cellulose, increase colonic mucus in rats with loperamide-induced constipation. Dig Dis Sci 46:1482-1489. https://doi. org/10.1023/a:1010644021888

7. Parthasarathy G, Chen J, Chen X, Chia N, O'Connor HM, Wolf PG, Gaskins HR, Bharucha AE (2016) Relationship between microbiota of the colonic mucosa vs feces and symptoms, colonic transit, and methane production in female patients with chronic constipation. Gastroenterology 150:367379.e361. https://doi.org/10.1053/j.gastro.2015.10.005

8. Avelar Rodriguez D, Popov J, Ratcliffe EM, Toro Monjaraz EM (2021) Functional constipation and the gut microbiome in children: preclinical and clinical evidence. Front Pediatr 8:595531-595531. https://doi.org/10. 3389/fped.2020.595531

9. Tabbers MM, DiLorenzo C, Berger MY, Faure C, Langendam MW, Nurko S, Staiano A, Vandenplas Y, Benninga MA (2014) Evaluation and treatment of functional constipation in infants and children: evidence-based recommendations from ESPGHAN and NASPGHAN. J Pediatr Gastroenterol Nutr 58:258-274. https://doi.org/10.1097/mpg.0000000000000266

10. Closa-Monasterolo R, Ferré N, Castillejo-DeVillasante G, Luque V, GispertLlaurado M, Zaragoza-Jordana M, Theis S, Escribano J (2017) The use of inulin-type fructans improves stool consistency in constipated children. A randomised clinical trial: pilot study. Int J Food Sci Nutr 68:587-594. https://doi.org/10.1080/09637486.2016.1263605

11. Polymeros D, Beintaris I, Gaglia A, Karamanolis G, Papanikolaou IS, Dimitriadis G, Triantafyllou K (2014) Partially hydrolyzed guar gum accelerates colonic transit time and improves symptoms in adults with chronic constipation. Dig Dis Sci 59:2207-2214. https://doi.org/10.1007/ s10620-014-3135-1

12. Üstündağ G, Kuloğlu Z, Kirbaş N, Kansu A (2010) Can partially hydrolyzed guar gum be an alternative to lactulose in treatment of childhood constipation? Turk J Gastroenterol 21:360-364. https://doi.org/10.4318/tjg.2010. 0121

13. Staiano A, Simeone D, Del Giudice E, Miele E, Tozzi A, Toraldo C (2000) Effect of the dietary fiber glucomannan on chronic constipation in neurologically impaired children. J Pediatr 136:41-45. https://doi.org/10. 1016/s0022-3476(00)90047-7

14. Loening-Baucke V, Miele E, Staiano A (2004) Fiber (glucomannan) is beneficial in the treatment of childhood constipation. Pediatrics 113:e259264. https://doi.org/10.1542/peds.113.3.e259

15. Beleli CA, Antonio MA, dos Santos R, Pastore GM, Lomazi EA (2015) Effect of $4^{\prime}$ galactooligosaccharide on constipation symptoms. Jornal de pediatria 91:567-573. https://doi.org/10.1016/j.jped.2015.01.010

16. Suares NC, Ford AC (2011) Systematic review: the effects of fibre in the management of chronic idiopathic constipation. Aliment Pharmacol Ther 33:895-901. https://doi.org/10.1111/j.1365-2036.2011.04602.x

17. Chen Z, Lin S, Jiang Y, Liu L, Jiang J, Chen S, Tong Y, Wang P (2019) Effects of bread yeast cell wall beta-glucans on mice with loperamide-induced constipation. J Med Food 22:1009-1021. https://doi.org/10.1089/jmf. 2019.4407

18. Żyła E, Dziendzikowska K, Gajewska M, Wilczak J, Harasym J, GromadzkaOstrowska J (2019) Beneficial effects of oat beta-glucan dietary supplementation in colitis depend on its molecular weight. Molecules. https:// doi.org/10.3390/molecules24193591 
19. Miyamoto J, Watanabe K, Taira S, Kasubuchi M, Li X, Irie J, Itoh H, Kimura I (2018) Barley $\beta$-glucan improves metabolic condition via short-chain fatty acids produced by gut microbial fermentation in high fat diet fed mice. PLoS ONE 13:e0196579. https://doi.org/10.1371/journal.pone.01965 79

20. Schwartz B, Hadar Y (2014) Possible mechanisms of action of mushroomderived glucans on inflammatory bowel disease and associated cancer. Ann Transl Med 2:19. https://doi.org/10.3978/j.issn.2305-5839.2014.01.03

21. Muthuramalingam $K$, Singh $V$, Choi $C$, Choi SI, Kim YM, Unno T, Cho M (2020) Dietary intervention using $(1,3) /(1,6)$ - $\beta$-glucan, a fungus-derived soluble prebiotic ameliorates high-fat diet-induced metabolic distress and alters beneficially the gut microbiota in mice model. Eur J Nutr 59:2617-2629. https://doi.org/10.1007/s00394-019-02110-5

22. Singh V, Muthuramalingam K, Kim YM, Park S, Kim SH, Lee J, Hyun C, Unno T, Cho M (2021) Synbiotic supplementation with prebiotic Schizophyllum commune derived $\beta-(1,3 / 1,6)$-glucan and probiotic concoction benefits gut microbiota and its associated metabolic activities. Appl Biol Chem 64:7. https://doi.org/10.1186/s13765-020-00572-4

23. Aron-Wisnewsky J, Prifti E, Belda E, Ichou F, Kayser BD, Dao MC, Verger EO, Hedjazi L, Bouillot J-L, Chevallier J-M, Pons N, Le Chatelier E, Levenez F, Ehrlich SD, Dore J, Zucker J-D, Clément K (2019) Major microbiota dysbiosis in severe obesity: fate after bariatric surgery. Gut 68:70-82. https://doi. org/10.1136/gutjnl-2018-316103

24. Dabke K, Hendrick G, Devkota S (2019) The gut microbiome and metabolic syndrome. J Clin Investig 129:4050-4057. https://doi.org/10.1172/ JCl129194

25. Parada Venegas D, De la Fuente MK, Landskron G, González MJ, Quera R, Dijkstra G, Harmsen HJM, Faber KN, Hermoso MA (2019) Short chain fatty acids (SCFAs)-mediated gut epithelial and immune regulation and its relevance for inflammatory bowel diseases. Front Immunol 10:277. https:// doi.org/10.3389/fimmu.2019.00277

26. Vu V, Muthuramalingam K, Singh V, Hyun C, Kim YM, Unno T, Cho M (2021) Effects of $\beta$-glucan, probiotics, and synbiotics on obesity-associated colitis and hepatic manifestations in C57BL/6J mice. Eur J Nutr. https://doi.org/10.1007/s00394-021-02668-z

27. Schloss PD, Westcott SL, Ryabin T, Hall JR, Hartmann M, Hollister EB, Lesniewski RA, Oakley BB, Parks DH, Robinson CJ, Sahl JW, Stres B, Thallinger GG, Horn DJV, Weber CF (2009) Introducing mothur: open-source, platform-independent, community-supported software for describing and comparing microbial communities. Appl Environ Microbiol 75:7537-7541. https://doi.org/10.1128/AEM.01541-09

28. Quast C, Pruesse E, Yilmaz P, Gerken J, Schweer T, Yarza P, Peplies J, Glöckner FO (2013) The SILVA ribosomal RNA gene database project: improved data processing and web-based tools. Nucleic Acids Res 41:D590-596. https://doi.org/10.1093/nar/gks1219

29. Rognes T, Flouri T, Nichols B, Quince C, Mahé F (2016) VSEARCH: a versatile open source tool for metagenomics. PeerJ 4:e2584. https://doi.org/10. 7717/peerj.2584

30. Cole JR, Wang Q, Fish JA, Chai B, McGarrell DM, Sun Y, Brown CT, PorrasAlfaro A, Kuske CR, Tiedje JM (2014) Ribosomal Database Project: data and tools for high throughput rRNA analysis. Nucleic Acids Res 42:D633-642. https://doi.org/10.1093/nar/gkt1244

31. Westcott SL, Schloss PD (2017) OptiClust, an improved method for assigning amplicon-based sequence data to operational taxonomic units. mSphere 2:e00073-e17. https://doi.org/10.1128/mSphereDirect.00073-17

32. Segata N, Izard J, Waldron L, Gevers D, Miropolsky L, Garrett WS, Huttenhower C (2011) Metagenomic biomarker discovery and explanation. Genome Biol 12:R60. https://doi.org/10.1186/gb-2011-12-6-r60

33. Douglas GM, Maffei VJ (2020) PICRUSt2 for prediction of metagenome functions. Nat Biotechnol 38:685-688. https://doi.org/10.1038/ s41587-020-0548-6

34. Parks DH, Beiko RG (2010) Identifying biologically relevant differences between metagenomic communities. Bioinformatics 26:715-721. https:// doi.org/10.1093/bioinformatics/bta041

35. Slavin J (2013) Fiber and prebiotics: mechanisms and health benefits. Nutrients 5:1417-1435. https://doi.org/10.3390/nu5041417

36. Mahdy Ali K, Wonnerth A, Huber K, Wojta J (2012) Cardiovascular disease risk reduction by raising $\mathrm{HDL}$ cholesterol-current therapies and future opportunities. Br J Pharmacol 167:1177-1194. https://doi.org/10.1111/j. 1476-5381.2012.02081.x
37. Kosmas CE, Martinez I, Sourlas A, Bouza KV, Campos FN, Torres V, Montan PD, Guzman E (2018) High-density lipoprotein (HDL) functionality and its relevance to atherosclerotic cardiovascular disease. Drugs in context 7:212525. https://doi.org/10.7573/dic.212525

38. Ho K-S, Tan CYM, MohdDaud MA, Seow-Choen F (2012) Stopping or reducing dietary fiber intake reduces constipation and its associated symptoms. World J Gastroenterol 18:4593-4596. https://doi.org/10.3748/ wjg.v18.i33.4593

39. Yang J, Wang H-P, Zhou L, Xu C-F (2012) Effect of dietary fiber on constipation: a meta analysis. World J Gastroenterol 18:7378-7383. https://doi. org/10.3748/wjg.v18.i48.7378

40. Fournier BM, Parkos CA (2012) The role of neutrophils during intestinal inflammation. Mucosal Immunol 5:354-366. https://doi.org/10.1038/mi. 2012.24

41. Ye J, Lv L, Wu W, Li Y, Shi D, Fang D, Guo F, Jiang H, Yan R, Ye W, Li L (2018) Butyrate protects mice against methionine-choline-deficient dietinduced non-alcoholic steatohepatitis by improving gut barrier function, attenuating inflammation and reducing endotoxin levels. Front Microbiol 9:1967. https://doi.org/10.3389/fmicb.2018.01967

42. Sato T, Matsumoto K, Okumura T, Yokoi W, Naito E, Yoshida Y, Nomoto K, Ito M, Sawada H (2008) Isolation of lactate-utilizing butyrate-producing bacteria from human feces and in vivo administration of Anaerostipes caccae strain L2 and galacto-oligosaccharides in a rat model. FEMS Microbiol Ecol 66:528-536. https://doi.org/10.1111/j.1574-6941.2008. 00528.x

43. Koh A, De Vadder F, Kovatcheva-Datchary P, Bäckhed F (2016) From dietary fiber to host physiology: short-chain fatty acids as key bacterial metabolites. Cell 165:1332-1345. https://doi.org/10.1016/j.cell.2016.05. 041

44. Cao H, Liu X, An Y, Zhou G, Liu Y, Xu M, Dong W, Wang S, Yan F, Jiang K, Wang B (2017) Dysbiosis contributes to chronic constipation development via regulation of serotonin transporter in the intestine. Sci Rep 7:10322. https://doi.org/10.1038/s41598-017-10835-8

45. Hawkes JS, Gibson RA, Roberton D, Makrides M (2006) Effect of dietary nucleotide supplementation on growth and immune function in term infants: a randomized controlled trial. Eur J Clin Nutr 60:254-264. https:// doi.org/10.1038/sj.ejcn.1602310

46. Dash NR, Al Bataineh MT (2021) Metagenomic analysis of the gut microbiome reveals enrichment of menaquinones (Vitamin K2) pathway in diabetes mellitus. Diabetes Metab J 45:77-85. https://doi.org/10.4093/ dmj.2019.0202

\section{Publisher's Note}

Springer Nature remains neutral with regard to jurisdictional claims in published maps and institutional affiliations.

\section{Submit your manuscript to a SpringerOpen ${ }^{\odot}$ journal and benefit from:}

- Convenient online submission

- Rigorous peer review

- Open access: articles freely available online

- High visibility within the field

- Retaining the copyright to your article

Submit your next manuscript at springeropen.com 